\title{
FORAMINA PARIETALIA PERMAGNA
}

\author{
KNud H. Krabhe
}

Le terme de foramen parietale, trou pariétal, a été employé pour deux formations différentes. En premier lieu, on dénomme foramina parietalia les deux trous des deux os pariétaux par lesquels les veines émissaires forment la connexion entre le sinus sagittal et les plexus veineux du galéa; ces trous sont situés très près de la fissure sagittale et assez rapprochés de la fissure lambdoïdale. En second lieu, le terme de foramen pariétal a été appliqué à la partie amincie du crâne au-dessus de l'œil pariétal rudimentaire chez les sauriens et quelques amphibiens.

Il s'agit de savoir s'il existe un rapport quelconque entre ces deux formations de trous perforant le crâne.

Les trous pariétaux qui contiennent les veines émissaires présentent quelquefois une dilatation symétrique extraordinaire. Nous avons eu l'occasion d'observer un cas de ce genre chez İequel on a pu constater à la radiographie et, du reste à la palpation, une dilatation énorme des trous pariétaux.

L'histoire du malade est sans importance pour la question des foramina pariétaux. Il faut simplement noter qu'il s'agissait d'un homme âgé de 4 th ans, facteur de profession, admis au Service Neurologique de Kommunehospitalet à Copenhague le 12 janvier 1952 et soigné sous les diagnostics de: myoses de la nuque, labyrinthose, céphalalgie histaminogène, foramina parietalia permagna. La radiographie $d u$ crâne montra ce qui suit: correspondant au siège des trous pariétaux, il y avait dans le crâne deux grandes défectuosités dans les os pariétaux. Ceiles-ci étaient rondes, symétriques, avaient un diamètre de deux centimètres environ. Élles étaient placées très près l'une de l'autre, de sorte qu'il semblait y avoir un très petit pont, ou plutôt un canal qui les reliait. Les bords des défectuosités étaient lisses (fig. 1, A). La radiographie ne donnait par ailleurs rien d'anormal; en particulier, il n'y avait aucune autre défectuosité du crâne, ni de soudures ouvertes. L'ossification des clavicules était normale.

A la palpation du crâne on observa, correspondant aux défectuosités du radiogramme, deux dépressions dans l'os, arrondies avec bords lisses. Le fond des deux dépressions était ferme comme une lame épaisse de tissu connectif, mais pas dure comme de l'os. La peau au-dessus des dépressions était normale, la chevelure de celle-ci également normale.

Le malade a six frères et sœurs, dont une sour présente la mème défectuosité du crâne (fig. 1, B). Du reste, pas de cas semblables dans la famille. La sour du malade, E. J., agée de 34 ans avait présenté de-même, depuis sa naissance, un "trou" dans la région parietale. La radiographie de son crâne montra un éclaircissement dans l'os pariétal, semblable à celui de son frère. Seulement il ètait un peu plus grand et les deux trous étaient fondus en un. L'anomalie n'avait jamais donné des gênes. 
Il est décrit dans la littérature cinquante cas environ de foramina pariétalia permagna. Un assez grand nombre de ces cas ont été décrits à des époques plus anciennes, alors que l'intérêt pour l'anatomie était plus
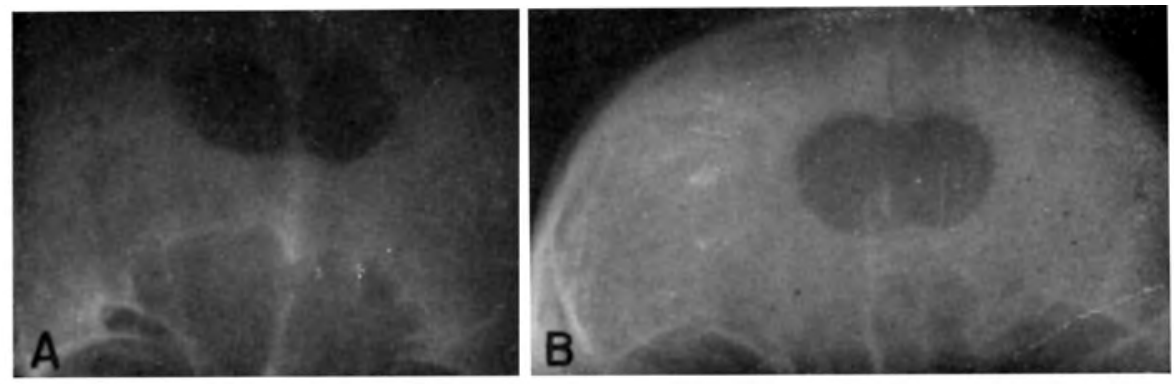

Fig. 1

marqué qu'à présent. Une des plus grandes collections de littérature se trouve dans un article de David Greig, dans l'Edinbourgh Medical Journal (1927).

Il convient d'ajouter quelques traits d'ordre anatomique normal. Si l'on examine le squelette d'un enfant nouveau-né, on voit qu'il y a, partant de la scissure sagittale, deux petites scissures transversales (fig. 2, A). Ce sont ces scissures qui sont l'issue des trous pariétaux. Il existe une certaine variabilité de cette partie. Nous allons citer ici ce qu'écrit TestutLatarjet sur cette variabilité:

"Lorsqu'on examine un crâne au 5ème mois de la vie fœetale, on s'aperçoit, tandis que les deux pariétaux sont à peu près en contact dans la plus grande partie de leur étendue, qu'il existe un point correspondant à la ligne de jonction des deux trous pariétaux, où les deux os sont encore séparés par un espace dont l'étendue, soit en longueur, atteint plusieurs centimètres. Que cet espace persiste après la naissance et l'on aura, en avant de la fontanelle postérieure, une deuxième fontanelle plus petite, c'est la fontanelle sagittale.

Signalée depuis longtemps par Gerdy et par Barkow, mais particulièrement bien étudiée, à une époque plus récente, par Hamy, par Broca et par Augier, la fontanelle sagittale siège à 2 centimètres en avant ỏu lambda, sur la suture des deux pariétaux. Elle peut être unilatérale, affectant alors la forme d'un petit triangle; ou bien elle empiète à la fois sur l'un et l'autre des pariétaux d'une façon symétrique et, dans ce cas, elle revêt une forme losangique. Les dimensions de la fontanelle sagittale varient de 1 à 30 millimètres. On la rencontrerait, d'après Broca, une fois sur quatre, sur les enfants nouveau-nés.

Le travail d'ossification tendant à combler la fontanelle sagittale par ses bords, il arrive un moment où cette lacune interpariétale n'est plus re- 


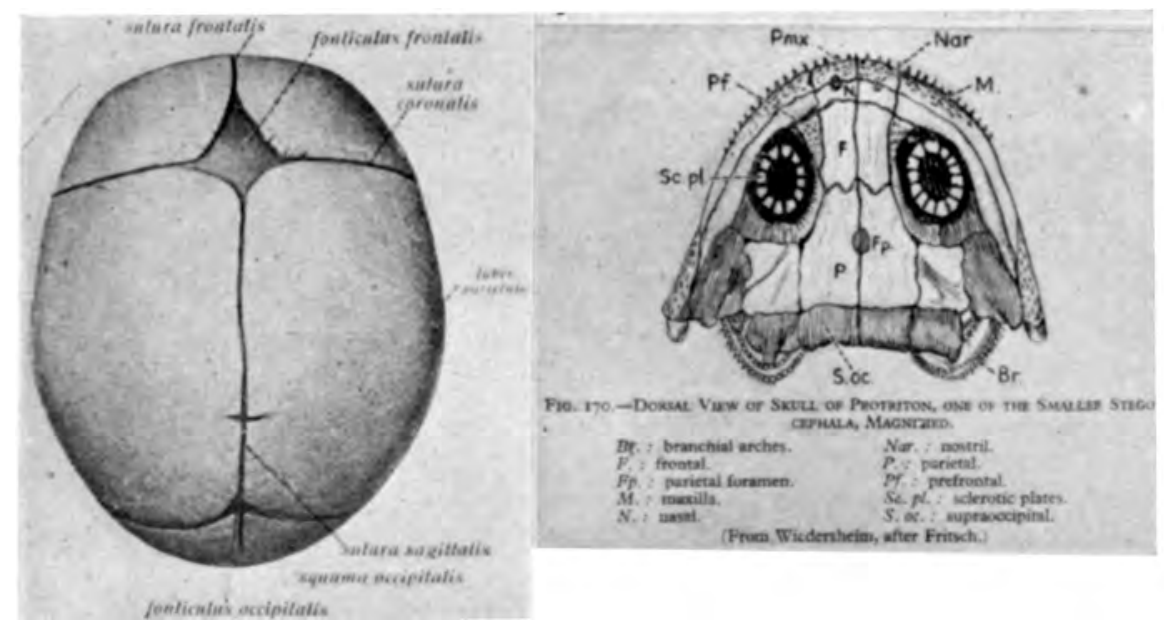

Fig. 2

présentée que par une simple scissure, à direction transversale, qui peut persister chez l'adulte. Ainsi se trouve constituée une anomalie nouvelle, l'incisure pariétale.

Les dimensions du trou pariétal varient, d'ordinaire, d'un tiers de millimètre à un millimètre. $\bar{A}$ la suite d'un trouble de l'ossification, on le voit atteindre de 4 à 5 millimètres et même davantage, 3 centimètres, la largeur du doigt. Quant au nombre des trous pariétaux, il est tout aussi variable: il n'en existe souvent qu'un seul, et, dans ce cas, le trou pariétal unique peut se trouver soit sur la ligne médiane, soit en dehors de cette ligne, à gauche ou à droite. Il peut y en avoir trois, deux d'un côté et un seul du côté opposé. Il peut, enfin, en exister quatre, deux de chaque côté.

Il semblerait résulter des observations de Broca que les trous pariétaux, quel qu'en soit le nombre, ne sont que de simples vestiges d'une scissure horizontale, l'incisure pariétale signalée ci-dessus, qui se serait fermée sur tous les autres points".

Nous allons examiner maintenant les trous pariétaux décrits chez les sauriens et les amphibiens actuels et éteints.

À l'état le plus marqué, on voit chez le Sphénodon punctatum, le seul présaurien existant maintenant, correspondant à l'œil pariétal relativement bien développé, un trou dans le crâne dans lequel l'œil est situé au-dessous de la peau transparente. Mais, même chez plusieurs sauriens il se trouve sur la ligne sagittale médiane un œil pariétal rudimentaire, développé à un degré variable. Chez les amphibiens urodèles l'œeil pariétal, encore plus rudimentaire, est placé hors du crâne, de sorte que la tige de 
l'œil, le nerf pariétal, traverse le trou pariétal. Et chez les saurierıs et les amphibiens, ce trou pariétal correspond à la fontanelle antérieure entre les os frontaux et les os pariétaux Cetie postition ne correspond pas à celle des foramina pariétalia chez l'homme.

Cependant, si l'on considère le trou pariélal chez les sauriens et amphibiens éteints, la situation est toui autre. On voit que le trou pariétal est souvent placé beaucoup plus en arrière, entre les deux os pariétaux. La figure 2, A montre le crâne d'un stégocéphale chez lequel le trou pariétal est situé presque au même endroit que la fontanelle interpariétale chez l'homme. Et l'on observe le même phénomène chez beaucoup d'autres.

Maintenant, il faut considérer les faits suivants: 1) Les trous pariétaux chez l'homme sont les seuls trous pour veines émissaires qui présentent dans quelques états pathologiques une dilatation énorme; 2) Les trous pariétaux chez l'homme sont développés dans la vie embryonnaire en connexion avec des scissures sortant de la scissure sagittale; 3) $\dot{A}$ cet endroit, il y a parfois une dilatation de la scissure sagittale chez l'homme, dénommée fontanelle interpariétale; 4) Presque au même endroit, entre les deux os pariétaux, on trouve chez les stégocéphales, peut-être les ancêtres éloignés des mammifères de notre temps, un trou pariétal correspondant à l'œil pariéíal rudimentaire (fig. 2, B); 5) Ces faits indiquent avec une certaine probabilité que la partie d'où se développent les foramina pariétalia chez l'homme est homologue au trou pariétal chez les stégocéphales.

Je sais très bien que les faits mentionnés ne prouvent pas que ces deux formations sont homologues; de tels phénomènes ne peuvent être prouvés. Il convient de voir s'il y a d'autres explications à l'existence de ces deux trous du crâne et s'il peut-être question de diagnostic différentiel.

Selon moi, il n'y a que deux phénomènes à considérer.

L'un est la maladie de Hand-Christian-Schüller; dans cette maladie on observe, surtout à la radiographie, des trous ronds dans le crâne. Cependant, dans cette maladie qui appartient aux lipoïdoses, les trous sont dispersés irrégulièrement dans les ou du crâne; ils ne sont pas placés de manière symétrique correspondant tout à fait aux trous pariétaux normaux. De plus, la maladie de Hand-Christian-Schüller est une maladie progressive, présentant beaucoup d'autres symptômes de nature très grave. Chez notre malade, ainsi que dans les cas décrits auparavant dans la littérature, les défectuosités ont été stationnaires depuis l'enfance, sans donner de symptômes pathologiques.

L'autrc maladie ou plutôt défectuosité qu'il faut prendre en considération est celle de la dysostose cléïdo-cranienne, dans laquelle on trouve des défectuosités dans l'ossification du crâne, ainsi que dans celle d'autres os, surtout les clavicules. Ce manque d'ossification peut-être rudimen- 
taire; par exemple, on observe des cas où l'ossification des clavicules est très restreinte. Mais même dans ce cas, on observe de petites défectuosités dans l'ossification claviculaire et d'autres traits, par exemple formation d'os wormiens.

Dans les cas de foramina pariétalia permagna, rien n'indique une connexion avec la dysostose cléïdo-cranienne.

Mais dans la grande collection des formations pathologiques chez l'homme qui semblent avoir une certaine connexion avec les organes rudimentaires - par exemple, les cystes branchiaux sur le cou, les côtes cervicales, les cystes paraphysaires du cerveau, les tumeurs parovaires - les fo. ramina pariétalia permagna doivent être considérés comme un phénomène qui a son point de départ dans le trou pariétal qui permettait à l'œil pariétal chez des ancêtres éloignés de l'homme, d'être un organe véritablement visuel.

\section{LITTERATURE}

David Greig (Edinburgh Medical Journal, New Series, vol. 34, 1927) donne une liste des articles sur les foramina parietalia permag̣na jusqu'à 1927. Dans le Quarterly Cumulative Index Medicus on va trouver, pour les ans suivants, environ vingt cinq indications d'articles sur ce thème. On les trouve sous les termes foramen et pariétal.

C, Dronningens Twaergade - Copenhagen $K$, Denmark (Dinamarca). 